\title{
Correction to: Normative values of the Rao's Brief Repeatable Battery in an Italian young adolescent population: the influence of age, gender, and education
} Fabrizia Falco ${ }^{1} \cdot$ Marcello Moccia $^{1}$ (D) Alessandro Chiodi ${ }^{2} \cdot$ Antonio Carotenuto $^{1} \cdot$ Angelo D'Amelio $^{2} \cdot$ Laura Rosa $^{3}$.
Kyrie Piscopo $^{3} \cdot$ Andrea Falco $^{1} \cdot$ Teresa Costabile $^{1} \cdot$ Francesca Lauro $^{1} \cdot$ Vincenzo Brescia Morra $^{1} \cdot$ Roberta Lanzillo $^{1}$

Published online: 5 April 2019

(C) Fondazione Società Italiana di Neurologia 2019

Correction to: Neurological Sciences 2019.

https://doi.org/10.1007/s10072-019-3712-3

The published version of this article unfortunately contained a mistake in Table 2. A wrong value on Table 2 under the heading 5th percentile to WLG is 15.00 and not 5.00. The Table is corrected here.

The online version of the original article can be found at https://doi.org/ 10.1007/s10072-019-3712-3

Marcello Moccia

moccia.marcello@gmail.com

1 Multiple Sclerosis Clinical Care and Research Centre, Department of Neuroscience, Reproductive Science and Odontostomatology,

Federico II University, Via Sergio Pansini, 5 - Building 17, Ground floor, Naples, Italy

2 Clinical Psychology Unit, Department of Neuroscience, Reproductive Science and Odontostomatology, Federico II University, Naples, Italy

3 Active Inclusion and Student Participation Service, Federico II University of Naples, Naples, Italy 
Table 2 BRB raw and adjusted scores and proposed cut-off points. The table shows BRB raw scores and scores adjusted for age, gender, and education (as necessary), with $95 \%$ confidence intervals $(95 \% \mathrm{CI})$, and proposed cut-off points (at the lowest 5 th percentile of adjusted scores)

\begin{tabular}{llll}
\hline Test & Raw score \pm SD [95\% CI] & Adjusted score \pm SD [95\% CI] & 5th percentile \\
\hline SRT-LTS & $52.95 \pm 9.87[50.73-55.17]$ & - & 36.75 \\
SRT-CLTR & $44.84 \pm 12.41[42.05-47.63]$ & - & 25.50 \\
SRT-D & $9.86 \pm 1.87[8.97-9.77]$ & - & 7.00 \\
SPART & $24.29 \pm 4.56[23.26-25.31]$ & $20.90 \pm 4.35[19.93-21.89]$ & 14.36 \\
SPART-D & $8.76 \pm 1.82[8.35-9.17]$ & $7.36 \pm 1.75[6.97-7.75]$ & 3.99 \\
SDMT & $59.80 \pm 11.21[57.28-62.32]$ & - & 41.50 \\
PASAT 3 & $43.42 \pm 10.33[41.10-45.74]$ & $40.35 \pm 9.73[31.17-42.55]$ & 24.27 \\
PASAT 2 & $35.83 \pm 10.85[33.39-38.27]$ & $30.76 \pm 9.68[28.58-32.94]$ & 15.80 \\
WLG & $21.33 \pm 4.74[20.26-22.40]$ & - & 15.00 \\
\hline
\end{tabular}

$B R B$, brief repeatable battery; SRT-LTS, selective reminding test long-term storage; SRT-CLTS, SRT consistent retrieval; SRT-D, SRT delayed recall; SPART, spatial recall test; SPART-D, SPART delayed recall; SDMT, symbol digit modalities test; PASAT, paced auditory serial addition test at 3 and $2 \mathrm{~s}$; $W L G$, word list generation; $S D$, standard deviation; $95 \%$ CI, 95\% confidence intervals

Publisher's note Springer Nature remains neutral with regard to jurisdictional claims in published maps and institutional affiliations. 